\title{
Bifurcation from interval and positive solutions for a class of fourth-order two-point boundary value problem
}

Wenguo Shen ${ }^{*}$ and Tao He

"Correspondence:

shenwg1963@126.com Department of Basic Courses, Lanzhou Institute of Technology, Lanzhou, 730050, People's Republic of China

\begin{abstract}
We consider the fourth-order two-point boundary value problem $x^{\prime \prime \prime \prime \prime}+k x^{\prime \prime}+I x=f(t, x), 0<t<1, x(0)=x(1)=x^{\prime}(0)=x^{\prime}(1)=0$, which is not necessarily linearizable. We give conditions on the parameters $k, /$ and $f(t, x)$ that guarantee the existence of positive solutions. The proof of our main result is based upon topological degree theory and global bifurcation techniques.
\end{abstract}

MSC: $34 \mathrm{~B} 15$

Keywords: topological degree; fourth-order ordinary differential equation; bifurcation; positive solution; eigenvalue

\section{Introduction}

The deformations of an elastic beam in an equilibrium state with fixed both endpoints can be described by the fourth-order boundary value problem

$$
\begin{aligned}
& x^{\prime \prime \prime \prime}+l x=\lambda h(t) f(x), \quad 0<t<1, \\
& x(0)=x(1)=x^{\prime}(0)=x^{\prime}(1)=0,
\end{aligned}
$$

where $f: \mathbb{R} \rightarrow \mathbb{R}$ is continuous, $\lambda \in \mathbb{R}$ is a parameter and $l$ is a given constant. Since problem (1.1) cannot transform into a system of second-order equations, the treatment method of the second-order system does not apply to it. Thus, the existing literature on problem (1.1) is limited. When $l=0$, the existence of positive solutions of problem (1.1) has been studied by several authors, see [1-5]. Especially, when $l \neq 0, \mathrm{Xu}$ and Han [6] studied the existence of nodal solutions of problem (1.1) by applying disconjugate operator theory and bifurcation techniques.

Recently, motivated by [6], when $k, l$ satisfy (A1), Shen [7] studied the existence of nodal solutions of a general fourth-order boundary value problem by applying disconjugate operator theory $[8,9]$ and Rabinowitz's global bifurcation theorem

$$
\begin{aligned}
& x^{\prime \prime \prime \prime}+k x^{\prime \prime}+l x=f(t, x), \quad 0<t<1, \\
& x(0)=x(1)=x^{\prime}(0)=x^{\prime}(1)=0,
\end{aligned}
$$

where

( 2013 Shen and He; licensee Springer. This is an Open Access article distributed under the terms of the Creative Commons Attribution License (http://creativecommons.org/licenses/by/2.0), which permits unrestricted use, distribution, and reproduction in any medium, provided the original work is properly cited. 
(A1) one of following conditions holds:

(i) $k, l$ satisfying $(k, l) \in\{(k, l) \mid k \in(-\infty, 0], l \in(0, \infty)\} \backslash\left\{\left(0, \frac{\pi^{4}}{64}\right)\right\} \cup\{(k, l) \mid k \in$ $\left.\left(-\infty, \pi^{2}\right), l \in(-\infty, 0]\right\}$ are given constants with

$$
\pi^{2}\left(k-\pi^{2}\right)<l \leq \frac{1}{4}\left(k-\frac{\pi^{2}}{4}\right)^{2}
$$

(ii) $k, l$ satisfying $(k, l) \in\left\{(k, l) \mid k \in\left(0, \frac{\pi^{2}}{2}\right), l \in(0, \infty)\right\}$ are given constants with

$$
\frac{1}{4}\left(\pi^{2} k-\frac{\pi^{4}}{4}\right)<l \leq \frac{1}{4} k^{2}
$$

In this paper, we consider bifurcation from interval and positive solutions for problem (1.2). In order to prove our main result, condition (A1) and the following weaker conditions are satisfied throughout this paper:

(H1) $f:[0,1] \times[0, \infty) \rightarrow[0, \infty)$ is continuous and there exist functions $a_{0}(t), a^{0}(t)$, $b_{\infty}(t)$, and $b^{\infty}(t) \in C([0,1],[0, \infty))$ such that

$$
a_{0}(t) x-\xi_{1}(t, x) \leq f(t, x) \leq a^{0}(t) x+\xi_{2}(t, x)
$$

for some functions $\xi_{1}, \xi_{2}$ defined on $[0,1] \times[0, \infty)$ with

$$
\xi_{1}(t, x)=o(x), \quad \xi_{2}(t, x)=o(x) \quad \text { as } x \rightarrow 0
$$

uniformly for $t \in[0,1]$, and

$$
b_{\infty}(t) x-\zeta_{1}(t, x) \leq f(t, x) \leq b^{\infty}(t) x+\zeta_{2}(t, x)
$$

for some functions $\zeta_{1}, \zeta_{2}$ defined on $[0,1] \times[0, \infty)$ with

$$
\zeta_{1}(t, x)=o(x), \quad \zeta_{2}(t, x)=o(x) \quad \text { as } x \rightarrow \infty
$$

uniformly for $t \in[0,1]$.

(H2) $f(t, x)>0$ for $t \in[0,1]$ and $x \in(0, \infty)$.

(H3) There exists a function $c(t) \in C([0,1],[0, \infty))$ with $c(t) \not \equiv 0$ in any subinterval of $[0,1]$ such that

$$
f(t, x) \geq c(t) x, \quad(t, x) \in[0,1] \times[0, \infty) .
$$

It is the purpose of this paper to study the existence of positive solutions of (1.2) under conditions (A1), (H1), (H2) and (H3). The main tool we use is the following global bifurcation theorem for the problem which is not necessarily linearizable.

Theorem A (Rabinowitz [10]) Let $V$ be a real reflexive Banach space. Let $F: \mathbb{R} \times V \rightarrow V$ be completely continuous such that $F(\lambda, 0)=0, \forall \lambda \in \mathbb{R}$. Let $a, b \in \mathbb{R}(a<b)$ be such that $u=0$ is an isolated solution of the following equation:

$$
u-F(\lambda, u)=0, \quad u \in V
$$


for $\lambda=a$ and $\lambda=b$, where $(a, 0),(b, 0)$ are not bifurcation points of (1.10). Furthermore, assume that

$$
d\left(I-F(a, \cdot), B_{r}(0), 0\right) \neq d\left(I-F(b, \cdot), B_{r}(0), 0\right),
$$

where $B_{r}(0)$ is an isolating neighborhood of the trivial solution. Let

$$
\mathcal{S}=\overline{\{(\lambda, u):(\lambda, u) \text { is a solution of }(1.10) \text { with } u \neq 0\}} \cup([a, b] \times\{0\}) .
$$

Then there exists a continuum (i.e., a closed connected set) $\mathcal{C}$ of $\mathcal{S}$ containing $[a, b] \times\{0\}$, and either

(i) $\mathcal{C}$ is unbounded in $V \times \mathbb{R}$, or

(ii) $\mathcal{C} \cap[(\mathbb{R} \backslash[a, b]) \times\{0\}] \neq \emptyset$.

Remark 1.1 For other results on the existence and multiplicity of positive solutions and nodal solutions for boundary value problems of fourth-order ordinary differential equations based on bifurcation techniques, see [11-20].

\section{Hypotheses and lemmas}

Let

$$
L[x]:=x^{\prime \prime \prime \prime}+k x^{\prime \prime}+l x .
$$

Theorem 2.1 (see [7, Theorem 2.4]) Let (A1) hold. Then

(i) $L[x]=0$ is disconjugate on $[0,1]$, and $L[x]$ has a factorization

$$
L[x]:=\rho_{4}\left(\rho_{3}\left(\rho_{2}\left(\rho_{1}\left(\rho_{0} x\right)^{\prime}\right)^{\prime}\right)^{\prime}\right)^{\prime},
$$

where $\rho_{k} \in C^{4-k}[0,1]$ with $\rho_{k}>0(k=0,1,2,3,4)$;

(ii) $x(0)=x(1)=x^{\prime}(0)=x^{\prime}(1)=0$ if and only if

$$
\left(L_{0} x\right)(0)=\left(L_{0} x\right)(1)=\left(L_{1} x\right)(0)=\left(L_{1} x\right)(1)=0,
$$

where

$$
\begin{aligned}
L_{0} x & =\rho_{0} x, \\
L_{i} x & =\rho_{i}\left(L_{i-1} x\right)^{\prime}, \quad i=1,2,3,4 .
\end{aligned}
$$

Theorem 2.2 (see [7, Theorem 2.7]) Let (A1) hold and $h \in C([0,1],[0, \infty))$ with $h(t) \not \equiv 0$ on any subinterval of $[0,1]$. Then

(i) the problem

$$
\left\{\begin{array}{l}
x^{\prime \prime \prime \prime}(t)+k x^{\prime \prime}(t)+l x(t)=\lambda h(t) x, \quad 0<t<1 \\
x(0)=x(1)=x^{\prime}(0)=x^{\prime}(1)=0
\end{array}\right.
$$

has an infinite sequence of positive eigenvalues

$$
0<\lambda_{1}(h)<\lambda_{2}(h)<\cdots<\lambda_{k}(h)<\lambda_{k+1}(h)<\cdots ;
$$


(ii) $\lambda_{k}(h) \rightarrow+\infty$ as $k \rightarrow+\infty$;

(iii) to each eigenvalue $\lambda_{k}(h)$, there corresponds an essential unique eigenfunction $\psi_{k}$ which has exactly $k-1$ simple zeros in $(0,1)$ and is positive near 0 ;

(iv) given an arbitrary subinterval of $[0,1]$, an eigenfunction that belongs to a sufficiently large eigenvalue changes its sign in that subinterval;

(v) for each $k \in \mathbb{N}$, the algebraic multiplicity of $\lambda_{k}(h)$ is 1 .

Theorem 2.3 (see [7, Theorem 2.8]) (Maximum principle) Let (A1) hold. Let $e \in C[0,1]$ with $e \geq 0$ on $[0,1]$ and $e \not \equiv$ in $[0,1]$. If $x \in C^{4}[0,1]$ satisfies

$$
\left\{\begin{array}{l}
x^{\prime \prime \prime \prime}(t)+k x^{\prime \prime}(t)+l x=e(t), \quad 0<t<1, \\
x(0)=x(1)=x^{\prime}(0)=x^{\prime}(1)=0,
\end{array}\right.
$$

then $x>0$ on $(0,1)$.

Let $Y=C[0,1]$ with the norm $\|x\|_{\infty}=\max _{t \in[0,1]}|x|$. Let $E=C^{2}[0,1]$ with its usual norm $\|x\|=\max \left\{\|x\|_{\infty},\left\|x^{\prime}\right\|_{\infty},\left\|x^{\prime \prime}\right\|_{\infty}\right\}$. By a positive solution of (1.2), we mean $x$ is a solution of (1.2) with $x>0$ (i.e., $x \geq 0$ in $(0,1)$ and $x \neq \equiv$ ).

Let $H:=L^{2}(0,1)$ with the inner product $\langle\cdot, \cdot\rangle$ and the norm $\|\cdot\|_{L^{2}}$. Further, define the linear operator $\widehat{L}: D(\widehat{L}) \subset E \rightarrow Y$

$$
\widehat{L} x=x^{\prime \prime \prime \prime}+k x^{\prime \prime}+l x, \quad x \in D(\widehat{L})
$$

with

$$
D(\widehat{L})=\left\{x \in C^{4}[0,1] \mid x(0)=x(1)=x^{\prime}(0)=x^{\prime}(1)=0\right\} .
$$

Then $\widehat{L}$ is a closed operator and $\widehat{L}^{-1}: Y \rightarrow E$ is completely continuous.

Lemma 2.4 Let $\psi_{1}$ be the first eigenfunction of (2.5). Then, for all $x \in D(\widehat{L})$, we get

$$
\left.\widehat{L} x, \psi_{1}\right\rangle=\left\langle x, \widehat{L} \psi_{1}\right\rangle .
$$

Proof Obviously, $\forall x \in D(\widehat{L})$, we have

$$
\psi_{1}(0)=\psi_{1}(1)=\psi_{1}^{\prime}(0)=\psi_{1}^{\prime}(1)=0, \quad x(0)=x(1)=x^{\prime}(0)=x^{\prime}(1)=0 .
$$

Integrating by parts, we obtain

$$
\begin{aligned}
\left\langle\widehat{L} x, \psi_{1}\right\rangle & =\int_{0}^{1}\left[x^{\prime \prime \prime \prime}(t)+k x^{\prime \prime}(t)+l x(t)\right] \psi_{1}(t) d t \\
& =\int_{0}^{1} x(t)\left[\psi_{1}^{\prime \prime \prime \prime}(t)+k \psi_{1}^{\prime \prime}(t)+l \psi_{1}(t)\right] d t=\left\langle x, \widehat{L} \psi_{1}\right\rangle .
\end{aligned}
$$

Let $\Sigma \subset \mathbb{R}^{+} \times E$ be the closure of the set of positive solutions of the problem

$$
\widehat{L} x=\lambda f(t, x) .
$$


We extend the function $f$ to a continuous function $\bar{f}$ defined on $[0,1] \times \mathbb{R}$ by

$$
\bar{f}(t, x)= \begin{cases}f(t, x), & (t, x) \in[0,1] \times[0, \infty], \\ f(t, 0), & (t, x) \in[0,1] \times(-\infty, 0] .\end{cases}
$$

Then $\bar{f}(t, x) \geq 0$ for $(t, x) \in[0,1] \times \mathbb{R}$. For $\lambda \geq 0$, let $x$ be an arbitrary solution of the problem

$$
\widehat{L} x=\lambda \bar{f}(t, x) .
$$

Since $\lambda \bar{f}(t, x(t)) \geq 0$ for $t \in[0,1]$, we have $x \geq 0$ for $t \in[0,1]$. Thus $x$ is a nonnegative solution of (2.11), and the closure of the set of nontrivial solutions $(\lambda, x)$ of $(2.13)$ in $\mathbb{R}^{+} \times E$ is exactly $\Sigma$.

Let $N: E \rightarrow Y$ be the Nemytskii operator associated with the function $\bar{f}$

$$
N(x)(t)=\bar{f}(t, x), \quad x \in E .
$$

Then (2.13), with $\lambda \geq 0$, is equivalent to the operator equation

$$
x=\lambda \widehat{L}^{-1} N(x), \quad x \in E
$$

In the following, we shall apply the Leray-Schauder degree theory, mainly to the mapping $\Phi_{\lambda}: E \rightarrow E$,

$$
\Phi_{\lambda}(x)=x-\lambda \widehat{L}^{-1} N(x) .
$$

For $R>0$, let $B_{R}=\{x \in E:\|x\| \leq R\}$, and let $\operatorname{deg}\left(\Phi_{\lambda}, B_{R}, 0\right)$ denote the degree of $\Phi_{\lambda}$ on $B_{R}$ with respect to 0 .

Lemma 2.5 Let $\Lambda \subset \mathbb{R}^{+}$be a compact interval with $\left[\lambda_{1}\left(a^{0}\right), \lambda_{1}\left(a_{0}\right)\right] \cap \Lambda=\emptyset$. Then there exists a number $\delta_{1}>0$ with the property

$$
\Phi_{\lambda}(x) \neq 0, \quad \forall x \in E: 0<\|x\| \leq \delta_{1}, \forall \lambda \in \Lambda .
$$

Proof Suppose to the contrary that there exist sequences $\left\{\mu_{n}\right\} \subset \Lambda$ and $\left\{x_{n}\right\}$ in $E: \mu_{n} \rightarrow$ $\mu^{*} \in \Lambda, x_{n} \rightarrow 0$ in $E$, such that $\Phi_{\mu_{n}}\left(x_{n}\right)=0$ for all $n \in \mathbb{N}$, then $x_{n} \geq 0$ in $[0,1]$.

Set $y_{n}=x_{n} /\left\|x_{n}\right\|$. Then $L y_{n}=\mu_{n}\left\|x_{n}\right\|^{-1} N\left(x_{n}\right)=\mu_{n}\left\|x_{n}\right\|^{-1} f\left(t, x_{n}\right)$ and $\left\|y_{n}\right\|=1$. Now, from condition (H1), we have the following:

$$
a_{0}(t) x_{n}-\xi_{1}\left(t, x_{n}\right) \leq f\left(t, x_{n}\right) \leq a^{0}(t) x_{n}+\xi_{2}\left(t, x_{n}\right)
$$

and, accordingly,

$$
\mu_{n}\left(a_{0}(t) y_{n}-\frac{\xi_{1}\left(t, x_{n}\right)}{\left\|x_{n}\right\|}\right) \leq \mu_{n} \frac{f\left(t, x_{n}\right)}{\left\|x_{n}\right\|} \leq \mu_{n}\left(a^{0}(t) y_{n}+\frac{\xi_{2}\left(t, x_{n}\right)}{\left\|x_{n}\right\|}\right) .
$$


Let $\varphi^{0}$ and $\varphi_{0}$ denote the nonnegative eigenfunctions corresponding to $\lambda_{1}\left(a^{0}\right)$ and $\lambda_{1}\left(a_{0}\right)$, respectively. Then we have, from the first inequality in (2.19),

$$
\left\langle\mu_{n}\left(a_{0}(t) y_{n}-\frac{\xi_{1}\left(t, x_{n}\right)}{\left\|x_{n}\right\|}\right), \varphi_{0}\right\rangle \leq\left\langle\mu_{n} \frac{f\left(t, x_{n}\right)}{\left\|x_{n}\right\|}, \varphi_{0}\right\rangle=\left\langle\widehat{L} y_{n}, \varphi_{0}\right\rangle
$$

From Lemma 2.4, we have

$$
\left\langle\widehat{L} y_{n}, \varphi_{0}\right\rangle=\left\langle y_{n}, \widehat{L} \varphi_{0}\right\rangle=\lambda_{1}\left(a_{0}\right)\left\langle y_{n}, a_{0}(t) \varphi_{0}\right\rangle .
$$

Since $x_{n} \rightarrow 0$ in $E$, from (1.6) we have

$$
\frac{\xi_{1}\left(t, x_{n}\right)}{\left\|x_{n}\right\|} \rightarrow 0 \quad \text { as }\left\|x_{n}\right\| \rightarrow 0
$$

By the fact that $\left\|y_{n}\right\|=1$, we conclude that $y_{n} \rightarrow y$ in $E$. Thus,

$$
\left\langle y_{n}, a_{0}(t) \varphi_{0}\right\rangle \rightarrow\left\langle y, a_{0}(t) \varphi_{0}\right\rangle
$$

Combining this and (2.21) and letting $n \rightarrow \infty$ in (2.20), we get

$$
\left\langle\mu^{*} a_{0}(t) y, \varphi_{0}\right\rangle \leq \lambda_{1}\left(a_{0}\right)\left\langle a_{0}(t) \varphi_{0}, y\right\rangle
$$

and consequently

$$
\mu^{*} \leq \lambda_{1}\left(a_{0}\right)
$$

Similarly, we deduce from the second inequality in (2.19) that

$$
\lambda_{1}\left(a^{0}\right) \leq \mu^{*}
$$

Thus, $\lambda_{1}\left(a^{0}\right) \leq \mu^{*} \leq \lambda_{1}\left(a_{0}\right)$. This contradicts $\mu^{*} \in \Lambda$.

Corollary 2.6 For $\lambda \in\left(0, \lambda_{1}\left(a^{0}\right)\right)$ and $\delta \in\left(0, \delta_{1}\right), \operatorname{deg}\left(\Phi_{\lambda}, B_{\delta}, 0\right)=1$.

Proof Lemma 2.5, applied to the interval $\Lambda=[0, \lambda]$, guarantees the existence of $\delta_{1}>0$ such that for $\delta \in\left(0, \delta_{1}\right)$,

$$
x-\tau \lambda \widehat{L}^{-1} N(x) \neq 0, \quad x \in E: 0<\|x\| \leq \delta, \tau \in[0,1] .
$$

Hence, for any $\delta \in\left(0, \delta_{1}\right)$,

$$
\operatorname{deg}\left(\Phi_{\lambda}, B_{\delta}, 0\right)=\operatorname{deg}\left(I, B_{\delta}, 0\right)=1,
$$

which ends the proof. 
Lemma 2.7 Suppose $\lambda>\lambda_{1}\left(a_{0}\right)$. Then there exists $\delta_{2}>0$ such that $\forall x \in E$ with $0<\|x\| \leq \delta_{2}$, $\forall \tau \geq 0$,

$$
\Phi_{\lambda}(x) \neq \tau \varphi_{0},
$$

where $\varphi_{0}$ is the nonnegative eigenfunction corresponding to $\lambda_{1}\left(a_{0}\right)$.

Proof We assume to the contrary that there exist $\tau_{n} \geq 0$ and a sequence $\left\{x_{n}\right\}$, with $\left\|x_{n}\right\|>0$ and $\left\|x_{n}\right\| \rightarrow 0$ in $E$, such that $\Phi_{\lambda}\left(x_{n}\right)=\tau_{n} \varphi_{0}$ for all $n \in \mathbb{N}$. As

$$
\widehat{L} x_{n}=\lambda N\left(x_{n}\right)+\tau_{n} \lambda_{1}\left(a_{0}\right) a_{0}(t) \varphi_{0}
$$

and $\tau_{n} \lambda_{1}\left(a_{0}\right) a_{0}(t) \varphi_{0} \geq 0$ in $(0,1)$, it follows that

$$
x_{n} \geq 0, \quad t \in[0,1] .
$$

Notice that $x_{n} \in D(\hat{L})$ has a unique decomposition

$$
x_{n}=\omega_{n}+s_{n} \varphi_{0},
$$

where $s_{n} \in \mathbb{R}$ and $\left\langle\omega_{n}, a_{0}(t) \varphi_{0}\right\rangle=0$. Since $x_{n} \geq 0$ on $[0,1]$ and $\left\|x_{n}\right\| \geq 0$, we have from (2.32) that $s_{n}>0$.

Choose $\sigma>0$ such that

$$
\sigma<\frac{\lambda-\lambda_{1}\left(a_{0}\right)}{\lambda} .
$$

By $(\mathrm{H} 1)$, there exists $r_{1}>0$ such that

$$
\left|\xi_{1}(t, x)\right| \leq \sigma a_{0}(t) x, \quad t \in[0,1], x \in\left[0, r_{1}\right]
$$

Therefore, for $t \in[0,1], x \in\left[0, r_{1}\right]$,

$$
f(t, x) \geq a_{0}(t) x-\xi_{1}(t, x) \geq(1-\sigma) a_{0}(t) x .
$$

Since $\left\|x_{n}\right\| \rightarrow 0$, there exists $N^{*}>0$ such that

$$
0 \leq x_{n} \leq r_{1}, \quad \forall n \geq N^{*}
$$

and consequently

$$
f\left(t, x_{n}\right) \geq(1-\sigma) a_{0}(t) x_{n}, \quad \forall n \geq N^{*} .
$$

Applying Lemma 2.4 and (2.37), it follows that

$$
\begin{aligned}
s_{n} \lambda_{1}\left(a_{0}\right)\left\langle\varphi_{0}, a_{0}(t) \varphi_{0}\right\rangle & =\left\langle x_{n}, \widehat{L} \varphi_{0}\right\rangle=\left\langle\widehat{L} x_{n}, \varphi_{0}\right\rangle \\
& =\lambda\left\langle N\left(x_{n}\right), \varphi_{0}\right\rangle+\tau_{n} \lambda_{1}\left(a_{0}\right)\left\langle a_{0}(t) \varphi_{0}, \varphi_{0}\right\rangle
\end{aligned}
$$




$$
\begin{aligned}
& \geq \lambda\left\langle N\left(x_{n}\right), \varphi_{0}\right\rangle \geq \lambda\left\langle(1-\sigma) a_{0}(t) x_{n}, \varphi_{0}\right\rangle \\
& =\lambda\left\langle(1-\sigma) a_{0}(t) \varphi_{0}, x_{n}\right\rangle \\
& =\lambda(1-\sigma) s_{n}\left\langle a_{0}(t) \varphi_{0}, \varphi_{0}\right\rangle .
\end{aligned}
$$

Thus,

$$
\lambda_{1}\left(a_{0}\right) \geq \lambda(1-\sigma)
$$

This contradicts (2.33).

Corollary 2.8 For $\lambda>\lambda_{1}\left(a_{0}\right)$ and $\delta \in\left(0, \delta_{2}\right), \operatorname{deg}\left(\Phi_{\lambda}, B_{\delta}, 0\right)=0$.

Proof Let $0<\delta \leq \delta_{2}$, where $\delta_{2}$ is the number asserted in Lemma 2.7. As $\Phi_{\lambda}$ is bounded in $\bar{B}_{\delta}$, there exists $c>0$ such that $\Phi_{\lambda}(x) \neq c \varphi_{0}$ for all $x \in \bar{B}_{\delta}$. By Lemma 2.7, one has

$$
\Phi_{\lambda}(x) \neq \tau c \varphi_{0}, \quad x \in \partial B_{\delta}, \tau \in[0,1] .
$$

Hence

$$
\operatorname{deg}\left(\Phi_{\lambda}, B_{\delta}, 0\right)=\operatorname{deg}\left(\Phi_{\lambda}-c \varphi_{0}, B_{\delta}, 0\right)=0 .
$$

Now, using Theorem A, we may prove the following.

Proposition 2.9 $\left[\lambda_{1}\left(a^{0}\right), \lambda_{1}\left(a_{0}\right)\right]$ is a bifurcation interval from the trivial solution for (2.15). There exists an unbounded component $C$ of a positive solution of (2.15), which meets $\left[\lambda_{1}\left(a^{0}\right), \lambda_{1}\left(a_{0}\right)\right] \times\{0\}$. Moreover,

$$
C \cap\left[\left(\mathbb{R} \backslash\left[\lambda_{1}\left(a^{0}\right), \lambda_{1}\left(a_{0}\right)\right]\right) \times\{0\}\right]=\emptyset .
$$

Proof For fixed $n \in \mathbb{N}$ with $\lambda_{1}\left(a^{0}\right)-\frac{1}{n}>0$, let us take that $a_{n}=\lambda_{1}\left(a^{0}\right)-\frac{1}{n}, b_{n}=\lambda_{1}\left(a_{0}\right)+\frac{1}{n}$ and $\bar{\delta}=\min \left\{\delta_{1}, \delta_{2}\right\}$. It is easy to check that for $0<\delta<\bar{\delta}$, all of the conditions of Theorem A are satisfied. So, there exists a connected component $C_{n}$ of solutions of (2.15) containing $\left[a_{n}, b_{n}\right] \times\{0\}$, and either

(i) $C_{n}$ is unbounded, or

(ii) $C_{n} \cap\left[\left(\mathbb{R} \backslash\left[a_{n}, b_{n}\right]\right) \times\{0\}\right] \neq \emptyset$.

By Lemma 2.5, the case (ii) cannot occur. Thus $C_{n}$ is unbounded bifurcated from $\left[a_{n}, b_{n}\right] \times\{0\}$ in $\mathbb{R} \times E$. Furthermore, we have from Lemma 2.5 that for any closed interval $I \subset\left[a_{n}, b_{n}\right] \backslash\left[\lambda_{1}\left(a^{0}\right), \lambda_{1}\left(a_{0}\right)\right]$, if $x \in\{x \in E \mid(\lambda, x) \in \Sigma, \lambda \in I\}$, then $\|x\| \rightarrow 0$ in $E$ is impossible. So, $C_{n}$ must be bifurcated from $\left[\lambda_{1}\left(a^{0}\right), \lambda_{1}\left(a_{0}\right)\right] \times\{0\}$ in $\mathbb{R} \times E$.

\section{Main results}

Theorem 3.1 Let (A1), (H1), (H2), (H3) hold. Assume that either

$$
\lambda_{1}\left(b_{\infty}\right)<1<\lambda_{1}\left(a^{0}\right)
$$


or

$$
\lambda_{1}\left(a_{0}\right)<1<\lambda_{1}\left(b^{\infty}\right)
$$

then problem (1.2) has at least one positive solution.

Proof of Theorem 3.1 It is clear that any solution of $(2.15)$ of the form $(1, x)$ yields a solution $x$ of (1.2). We will show that $C$ crosses the hyperplane $\{1\} \times E$ in $\mathbb{R} \times E$. To do this, it is enough to show that $C$ joins $\left[\lambda_{1}\left(a^{0}\right), \lambda_{1}\left(a_{0}\right)\right] \times\{0\}$ to $\left[\lambda_{1}\left(b^{\infty}\right), \lambda_{1}\left(b_{\infty}\right)\right] \times\{\infty\}$. Let $\left(\mu_{n}, x_{n}\right) \in$ $C$ satisfy

$$
\mu_{n}+\left\|x_{n}\right\| \rightarrow \infty
$$

We note that $\mu_{n}>0$ for all $n \in \mathbb{N}$ since $(0,0)$ is the only solution of (2.15) for $\lambda=0$ and $C \cap(\{0\} \times E)=\emptyset$.

Case 1. $\lambda_{1}\left(b_{\infty}\right)<1<\lambda_{1}\left(a^{0}\right)$.

In this case, we show that

$$
\left(\lambda_{1}\left(b_{\infty}\right), \lambda_{1}\left(a^{0}\right)\right) \subseteq\{\lambda \in \mathbb{R} \mid(\lambda, x) \in C\}
$$

We divide the proof into two steps.

Step 1 . We show that $\left\{\mu_{n}\right\}$ is bounded.

Since $\left(\mu_{n}, x_{n}\right) \in C, L x_{n}=\mu_{n} f\left(t, x_{n}\right)$. From (H3), we have

$$
L x_{n} \geq \mu_{n} c(t) x_{n} .
$$

Let $\bar{\varphi}$ denote the nonnegative eigenfunction corresponding to $\lambda_{1}(c)$.

From (3.4), we have

$$
\left\langle L x_{n}, \bar{\varphi}\right\rangle \geq \mu_{n}\left\langle c(t) x_{n}, \bar{\varphi}\right\rangle \text {. }
$$

By Lemma 2.4, we have

$$
\lambda_{1}(c)\left\langle x_{n}, c(t) \bar{\varphi}\right\rangle=\left\langle x_{n}, L \bar{\varphi}\right\rangle \geq \mu_{n}\left\langle c(t) \bar{\varphi}, x_{n}\right\rangle
$$

Thus

$$
\mu_{n} \leq \lambda_{1}(c)
$$

Step 2. We show that $C$ joins $\left[\lambda_{1}\left(a^{0}\right), \lambda_{1}\left(a_{0}\right)\right] \times\{0\}$ to $\left[\lambda_{1}\left(b^{\infty}\right), \lambda_{1}\left(b_{\infty}\right)\right] \times\{\infty\}$.

From (3.3) and (3.7), we have that $\left\|x_{n}\right\| \rightarrow \infty$. Notice that (2.15) is equivalent to the integral equation

$$
x_{n}(t)=\mu_{n} \int_{0}^{1} G(t, s) f\left(s, x_{n}(s)\right) d s \text {, }
$$


which implies that

$$
\begin{aligned}
& \mu_{n} \int_{0}^{1} G(t, s)\left[b_{\infty}(s) x_{n}(s)-\zeta_{1}\left(s, x_{n}(s)\right)\right] d s \\
& \quad \leq x_{n}(t) \\
& \quad \leq \mu_{n} \int_{0}^{1} G(t, s)\left[b^{\infty}(s) x_{n}(s)+\zeta_{2}\left(s, x_{n}(s)\right)\right] d s .
\end{aligned}
$$

We divide both of (3.9) by $\left\|x_{n}\right\|$ and set $y_{n}=\frac{x_{n}}{\left\|x_{n}\right\|}$. Since $y_{n}$ is bounded in $E$, there exists a subsequence of $\left\{y_{n}\right\}$ and $y^{*} \in E$, with $y^{*} \geq 0$ and $y^{*} \not \equiv$ on $(0,1)$, such that

$$
\mu_{n} \rightarrow \mu^{*}, \quad y_{n} \rightarrow y^{*} \quad \text { in } E,
$$

relabeling if necessary. Thus, (3.9) yields that

$$
\mu^{*} \int_{0}^{1} G(t, s) b_{\infty}(s) y^{*}(s) d s \leq y^{*}(t) \leq \mu^{*} \int_{0}^{1} G(t, s) b^{\infty}(s) y^{*}(s) d s,
$$

which implies that

$$
\mu^{*} b_{\infty}(t) y^{*} \leq L y^{*} \leq \mu^{*} b^{\infty}(t) y^{*} \leq L y^{*} .
$$

Let $\varphi^{\infty}$ and $\varphi_{\infty}$ denote the nonnegative eigenfunction corresponding to $\lambda_{1}\left(b^{\infty}\right)$ and $\lambda_{1}\left(b_{\infty}\right)$, respectively. Then we have, from the first inequality in (3.12),

$$
\left\langle\mu^{*} b_{\infty}(t) y^{*}, \varphi_{\infty}\right\rangle \leq\left\langle L y^{*}, \varphi_{\infty}\right\rangle
$$

From Lemma 2.4, integrating by parts, we obtain that

$$
\mu^{*}\left\langle b_{\infty}(t) y^{*}, \varphi_{\infty}\right\rangle \leq\left\langle L y^{*}, \varphi_{\infty}\right\rangle=\left\langle L \varphi_{\infty}, y^{*}\right\rangle=\lambda_{1}\left(b_{\infty}\right)\left\langle b_{\infty}(t) \varphi_{\infty}, y^{*}\right\rangle,
$$

and consequently

$$
\mu^{*} \leq \lambda_{1}\left(b_{\infty}\right)
$$

Similarly, we deduce from the second inequality in (3.12) that

$$
\lambda_{1}\left(b^{\infty}\right) \leq \mu^{*} .
$$

Thus

$$
\lambda_{1}\left(b^{\infty}\right) \leq \mu^{*} \leq \lambda_{1}\left(b_{\infty}\right) .
$$

So, $C$ joins $\left[\lambda_{1}\left(a^{0}\right), \lambda_{1}\left(a_{0}\right)\right] \times\{0\}$ to $\left[\lambda_{1}\left(b^{\infty}\right), \lambda_{1}\left(b_{\infty}\right)\right] \times\{\infty\}$.

Case 2. $\lambda_{1}\left(a_{0}\right)<1<\lambda_{1}\left(b^{\infty}\right)$.

In this case, if $\left(\mu_{n}, x_{n}\right) \in C$ is such that

$$
\lim _{n \rightarrow \infty}\left(\mu_{n}+\left\|x_{n}\right\|\right)=\infty
$$


and

$$
\lim _{n \rightarrow \infty} \mu_{n}=\infty
$$

then

$$
\left(\lambda_{1}\left(a_{0}\right), \lambda_{1}\left(b^{\infty}\right)\right) \subseteq\{\lambda \in(0, \infty) \mid(\lambda, x) \in C\}
$$

and, moreover,

$$
(\{1\} \times E) \cap C \neq \emptyset .
$$

Assume that $\left\{\mu_{n}\right\}$ is bounded; applying a similar argument to that used in Step 2 of Case 1 , after taking a subsequence and relabeling if necessary, we obtain

$$
\mu_{n} \rightarrow \mu^{*} \in\left[\lambda_{1}\left(a_{0}\right), \lambda_{1}\left(b^{\infty}\right)\right], \quad\left\|x_{n}\right\| \rightarrow \infty \quad \text { as } n \rightarrow \infty
$$

Again $C$ joins $\left[\lambda_{1}\left(a^{0}\right), \lambda_{1}\left(a_{0}\right)\right] \times\{0\}$ to $\left[\lambda_{1}\left(b^{\infty}\right), \lambda_{1}\left(b_{\infty}\right)\right] \times\{\infty\}$ and the result follows.

\section{Competing interests}

The authors declare that they have no competing interests.

\section{Authors' contributions}

WS conceived of the study, and participated in its design and coordination and helped to draft the manuscript. TH drafted the manuscript. All authors read and approved the final manuscript.

\section{Acknowledgements}

This work is supported by the NSF of Gansu Province (No. 1114-04).

Received: 14 November 2012 Accepted: 8 July 2013 Published: 22 July 2013

\section{References}

1. Agarwal, RP, Chow, YM: Iterative methods for a fourth-order boundary value problem. J. Comput. Appl. Math. 10(2), 203-217 (1984). doi:10.1016/0377-0427(84)90058-X

2. Ma, R, Wu, HP: Positive solutions of a fourth-order two-point boundary value problem. Acta Math. Sci., Ser. A 22(2), 244-249 (2002)

3. Yao, Q: Positive solutions for eigenvalue problems of fourth-order elastic beam equations. Appl. Math. Lett. 17(2), 237-243 (2004). doi:10.1016/S0893-9659(04)90037-7

4. Yao, Q: Solvability of an elastic beam equation with Caratheodory function. Math. Appl. 17(3), 389-392 (2004)

5. Korman, P: Uniqueness and exact multiplicity of solutions for a class of fourth-order semilinear problems. Proc. R. Soc. Edinb. A 134(1), 179-190 (2004). doi:10.1017/S0308210500003140

6. Xu, J, Han, XL: Nodal solutions for a fourth-order two-point boundary value problem. Bound. Value Probl. 2010, Article ID 570932 (2010)

7. Shen, WG: Existence of nodal solutions of a nonlinear fourth-order two-point boundary value problem. Bound. Value Probl. (2012). doi:10.1186/1687-2770-2012-31

8. Elias, U: Eigenvalue problems for the equations $L y+\lambda p(x) y=0$. J. Differ. Equ. 29(1), 28-57 (1978). doi:10.1016/0022-0396(78)90039-6

9. Elias, U: Oscillation Theory of Two-Term Differential Equations. Mathematics and Its Applications, vol. 396. Kluwer Academic, Dordrecht (1997)

10. Rabinowitz, PH: Some aspects of nonlinear eigenvalue problems. Rocky Mt. J. Math. 3, 161-202 (1973)

11. Ma, R: Existence of positive solutions of a fourth-order boundary value problem. Appl. Math. Comput. 168(2), 1219-1231 (2005). doi:10.1016/j.amc.2004.10.014

12. $\mathrm{Ma}, \mathrm{R}$ : Nodal solutions for a fourth-order two-point boundary value problem. J. Math. Anal. Appl. 314(1), 254-265 (2006). doi:10.1016/j.jmaa.2005.03.078

13. Ma, R: Nodal solutions of boundary value problem of fourth-order ordinary differential equations. J. Math. Anal. Appl. 319(2), 424-434 (2006). doi:10.1016/j.jmaa.2005.06.045

14. Ma, R, Thompson, B: Nodal solutions for a nonlinear fourth-order eigenvalue problem. Acta Math. Sin. Engl. Ser. 24(1), 27-34 (2008). doi:10.1007/s10114-007-1009-6

15. $\mathrm{Ma}, \mathrm{R}, \mathrm{Xu}, \mathrm{J}$ : Bifurcation from interval and positive solutions of a fourth-order boundary value problem. Nonlinear Anal., Theory Methods Appl. 72(1), 113-122 (2010). doi:10.1016/j.na.2009.06.061 
16. Bai, Z, Wang, H: On positive solutions of some nonlinear fourth-order beam equations. J. Math. Anal. Appl. 270(1), 357-368 (2006). doi:10.1016/S0022-247X(02)00071-9

17. $\mathrm{Ma}, \mathrm{R}, \mathrm{GaO}, \mathrm{CH}, \mathrm{Han}, \mathrm{XL}$ : On linear and nonlinear fourth-order eigenvalue problems with indefinite weight. Nonlinear Anal., Theory Methods Appl. 74(18), 6965-6969 (2011). doi:10.1016/..na.2011.07.017

18. Ma, R, Gao, CH: Nodal solutions of a nonlinear eigenvalue problem of the Euler-Bernoulli equation. J. Math. Anal. Appl. 387(2), 1160-1166 (2012). doi:10.1016/j.jmaa.2011.10.019

19. Ma, R, Chen, TL: Existence of positive solutions of fourth-order problems with integral boundary conditions. Bound. Value Probl. 2011, Article ID 297578 (2011)

20. Ma, R, Xu, L: Existence of positive solutions of a nonlinear fourth-order boundary value problem. Appl. Math. Lett. 23(5), 537-543 (2010). doi:10.1016/j.aml.2010.01.007

doi:10.1186/1687-2770-2013-170

Cite this article as: Shen and He: Bifurcation from interval and positive solutions for a class of fourth-order two-point boundary value problem. Boundary Value Problems 2013 2013:170.

Submit your manuscript to a SpringerOpen ${ }^{\circ}$ journal and benefit from:

- Convenient online submission

- Rigorous peer review

- Immediate publication on acceptance

Open access: articles freely available online

- High visibility within the field

- Retaining the copyright to your article 